\title{
Brain Stimulation Reward is Integrated by A Network of Electrically-Coupled GABA Neurons
}

\author{
Matthew Brian Lassen \\ Brigham Young University - Provo
}

Follow this and additional works at: https://scholarsarchive.byu.edu/etd

Part of the Neuroscience and Neurobiology Commons

\section{BYU ScholarsArchive Citation}

Lassen, Matthew Brian, "Brain Stimulation Reward is Integrated by A Network of Electrically-Coupled GABA Neurons" (2006). Theses and Dissertations. 1298.

https://scholarsarchive.byu.edu/etd/1298

This Thesis is brought to you for free and open access by BYU ScholarsArchive. It has been accepted for inclusion in Theses and Dissertations by an authorized administrator of BYU ScholarsArchive. For more information, please contact scholarsarchive@byu.edu, ellen_amatangelo@byu.edu. 


\title{
BRAIN STIMULATION REWARD IS INTEGRATED BY A NETWORK OF ELECTRICALLY COUPLED GABA NEURONS
}

\author{
By \\ Matthew Brian Lassen
}

\begin{abstract}
A thesis submitted to the faculty of
Brigham Young University

in partial fulfillment of the requirements for the degree of

Master of Science
\end{abstract}

Department of Physiology and Developmental Biology

Brigham Young University

August 2006 
Copyright (C) 2006 Matthew Brian Lassen

All Rights Reserved 


\section{BRIGHAM YOUNG UNIVERSITY}

\section{GRADUATE COMMITTEE APPROVAL}

of a thesis submitted by

Matthew Brian Lassen

This prospectus has been read by each member of the following graduate committee and by majority vote has been found to be satisfactory.

Date

Date

Date
Scott C. Steffensen, Chair

Sterling Sudweeks 


\section{BRIGHAM YOUNG UNIVERSITY}

As chair of the candidate's graduate committee, I have read the thesis of Matthew Lassen in its final form and have found that (1) its format, citations, and bibliographical style are consistent and acceptable and fulfill university and department style requirements; (2) its illustrative materials including figures, tables, and charts are in place; and (3) the final manuscript is satisfactory to the graduate committee and is ready for submission to the university library.

Date

Accepted for the Department
Scott C. Steffensen

Chair, Graduate Committee

James Porter

Chair, PDBio

Accepted for the College

John D. Bell

Associate Dean, College of Biology and Agriculture 


\begin{abstract}
BRAIN STIMULATION REWARD IS INTEGRATED BY A NETWORK OF ELECTRICALLY-COUPLED GABA NEURONS

\author{
Matthew Brian Lassen \\ Department of Physiology and Developmental Biology \\ Master of Science: Neuroscience
}

\begin{abstract}
Although it is well-established that animals will self-stimulate electric current to various diverse brain structures, the neural substrate of brain stimulation reward (BSR) has eluded identification since its discovery more than a half-century ago. We show that GABA neurons in the midbrain, hypothalamus and thalamus express connexin-36 (Cx36) gap junctions and couple electrically with dopamine application or by stimulation of the internal capsule (IC), which also supports self-stimulation. The threshold for responding for self-stimulation of the IC is the threshold for coupling between these GABA neurons, the degree of responding for IC ICSS is proportional to the magnitude of electrical coupling between these GABA neurons, and GJ blockers, including the $\mathrm{Cx} 36$ blocker mefloquine, increase the threshold for IC self-stimulation without affecting performance. Thus, electrical coupling between this network of GABA neurons fits the prevailing model for the elusive integrator of BSR.
\end{abstract}




\section{ACKNOWLEDGEMENTS}

I wish to acknowledge the daily assistance and kind support of my mentor, Scott Steffensen. I would like to thank my wonderful and supportive wife Sarah Lassen (Stobbs), and also Seth Gunderson, Steve Davis, Katie Wilber, Mattheau Eysser, Stuart Thomas and the students of Neuroscience 481 for giving countless hours to this project. Thanks also to my parents, Gary and Bonny Lassen, for their love and support, which manifested itself in many ways. 
TABLE OF CONTENTS

THESIS.........................................................................

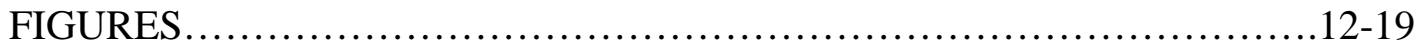

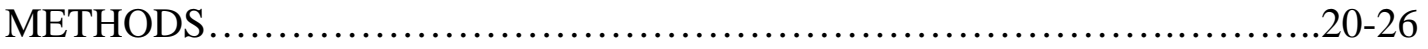

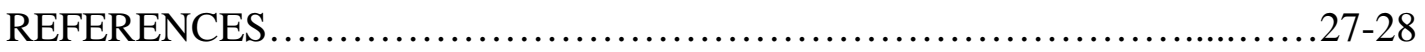

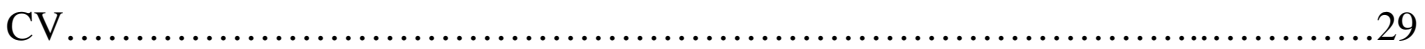




\section{TABLE OF FIGURES}

FIGURE 1 (Co-expression of Cx36 and GAD65,67 in the dorsal VTA)

FIGURE 2 (Co-expression of CX36 and GAD65,67 in structures along the rostrocaudal neuraxis from the ventral tegmental area (VTA) to the reticular

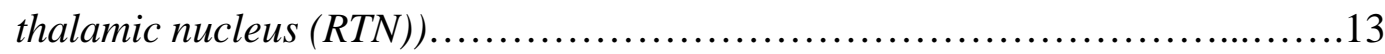

FIGURE 3 (Dopamine-sensitive electrically-coupled GABA neurons are localized to the VTA and nearby structures and correlate with areas showing

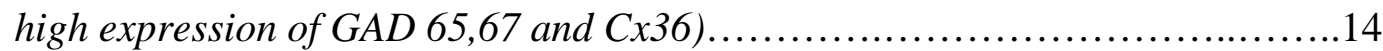

FIGURE 4 (Coupling of neurons along the rostrocaudal ventral neuraxis at locations demonstrating co-expression of Cx36 and GAD65,67 transcripts).......15

FIGURE 5 (The threshold for VTA GABA neuron ICPSDs is the threshold for IC selfstimulation responding)........................................................

FIGURE 6 (Gap junction blockers raise the threshold for IC self-stimulation responding.

FIGURE 7 (Input/output functions of IC-evoked post-stimulus spike discharges (ICPSDs): Monotonic function of pulse number and stimulus intensity). .18

FIGURE 8 (IC self-stimulation responding is a function of coupling between VTA GABA Neurons) 


\section{BRAIN STIMULATION REWARD IS INTEGRATED BY A NETWORK OF ELECTRICALLY-COUPLED GABA NEURONS}

Since the landmark report by Olds et al (1) the phenomenon of brain stimulation reward (BSR) via electrical self-stimulation has undergone considerable scrutiny. This obtains, in part, because of the expectancy that the neurophysiological substrates underlying BSR might elucidate the neuronal underpinnings of natural rewarding behaviors. Therefore, one of the primary objectives of research on BSR is the identification of the directly-stimulated neuronal substrate. However, uncovering the neural substrate or transducer of BSR has proven to be problematical as stimulation of multiple, often diverse, brain structures, as well as pathways with complex fiber systems, support BSR (for review see (2)). Notwithstanding the difficulties in identifying the primary transducer of BSR, pharmacological (3), neurochemical (4), and lesion studies (5) have implicated the mesocorticolimbic dopamine (DA) system originating in the ventral tegmental area (VTA) of the midbrain. However, psychophysical studies have determined that the primary transducer of BSR could not be mesencephalic DA neurons as the reward-related fibers directly activated by the selfstimulating electrode descend through the MFB while DA fibers ascend through the MFB (6) and have conduction velocities too low and refractory periods too high to account for the physiological evidence (7-11). Collectively, these conclusions have been known as the 'descending path hypothesis' (12-15). Nonetheless, pharmacological studies have shown that midbrain DA neurons are still considered to be involved in BSR, but may constitute second or third-order neurons in the mesocorticolimbic reward circuit (3). Moreover, although DA release is tonically elevated during BSR (4), there is no phasic release of NAcc DA in association with each response for BSR (16). But perhaps the greatest paradox is that very large lesions of the MFB often have very little effect on the psychophysically-measured magnitude of BSR $(5,17)$. Thus, while DA neurotransmission plays a role in the rewarding effect of MFB stimulation, the evidence is lacking for its primary role in BSR. It has been suggested that the primary neural substrate of BSR is a diffuse net-like connection between forebrain nuclei and the VTA (17). 
We have previously characterized a homogeneous population of midbrain nonDA neurons that contain GABA, and project to, and receive input from, corticolimbic structures (18), and have recently reported that they are coupled electrically in the mature brain via connexin-36 (Cx36) gap junctions (19), and their coupling is enhanced by DA or by stimulation of the internal capsule (IC; (20)). We have found that the discharge activity of these neurons is correlated with discrete movements and cortical arousal (21), and accelerates in anticipation of self-stimulation of the MFB (22). By virtue of their proximity to midbrain DA neurons, wide dynamic range, widespread axonal distribution, sensitivity to DA (20), their presumed synaptic connectivity to DA neurons, and their electrical coupling, midbrain GABA neurons may be critical integrators of mesocorticolimbic DA neurotransmission implicated in natural and drug reward. Here we present anatomical, physiological, pharmacological and behavioral data implicating these neurons, and the electrical GABA network they comprise, as the neuronal integrator of BSR. We will show anatomical and immunohistochemical evidence for the distribution of Cx36 GJs in GABA neurons in the VTA and other areas along the ventral brain neuraxis, and correlate this distribution with physiological evidence for electrical coupling. Using single-cell recording electrophysiological techniques, we will show physiological evidence demonstrating the input/output characteristics of ventral brain GABA neuron coupling via IC stimulation, the site used for self-stimulation. Finally, using the IC self-stimulation behavioral paradigm, we will show evidence that IC stimulation supports BSR, that the threshold for IC-induced coupling between these GABA neurons is the threshold for BSR, that the degree of coupling between these GABA neurons correlates with the degree of BSR, and that GJ blockers raise the threshold for BSR.

Cx36 GJs are readily detected in subsets of interneurons in many regions of the CNS $(23,24)$. We have recently demonstrated that dorsal VTA GABA neurons express Cx36 transcripts and protein (25). To determine the extent and distribution of Cx36 expressing GABA neurons in the VTA (Fig. 1A-C) we evaluated mRNA expression of GAD65,67, a marker of GABA neurons, and Cx36 with fluorescent in-situ 
hybridization (FISH). Low-power and high-power images of horizontal brain slices obtained from 4 rats at the level of the VTA revealed high-density co-expression of GAD65,67/Cx36 in neurons in the dorsal VTA (Fig. 1D; 66\% of the neurons were $\mathrm{Cx} 36+, 27 \%$ of the neurons were GAD65,67+, and $34 \%$ of the neurons were Cx36+/GAD65,67+; all neurons that were GAD65,67+ were Cx36+; $n=4)$. To confirm that the mRNA transcripts result in expression of these proteins we evaluated the expression of GAD65,67 and Cx36 protein in the dorsal VTA with immunohistochemistry. Low-power and high-power images of horizontal dorsal brain slices revealed co-expression of GAD65,67 and Cx36 protein in the dorsal VTA (Fig. 1E), albeit not at the levels of the co-expression of transcripts seen in the FISH images, perhaps a result of the subcellular differential compartmentalization of these two proteins (e.g., Cx36 in dendrodendritic GJs vs GAD65,67 in somata and terminals). While the dorsal VTA was characterized by high-density coexpression of $\mathrm{Cx} 36$ and GAD65,67, the ventral VTA was characterized by only low-density coexpression of these transcripts (Fig. 2A). In order to further evaluate the anatomical distribution of neurons co-expressing Cx36 and GAD65,67 we evaluated FISH images along the rostrocaudal neuraxis in horizontal brain slices at the level of the dorsal and ventral VTA. We found high-density coexpression of $\mathrm{Cx} 36$ and GAD65,67 transcripts in the substantia nigra reticulata ( $\mathrm{SNr}$ ), lateral hypothalamus $(\mathrm{LH})$, anterior hypothalamus (AH), medial preoptic area (MPA), medial preoptic nucleus (MPO), ventral hippocampus (HPC) and reticular thalamic nuclues (RTN; Fig. 2B-G).

We wanted to determine if electrical coupling between GABA neurons might occur in the dorsal VTA and in those regions of the rostrocaudal neuraxis containing a high-density of GAD65,67 and Cx36 co-expression. We have recently demonstrated that DA application or stimulation of the internal capsule (IC) induces electrical coupling between VTA GABA neurons ((19, 20); Fig. 3A-C). We evaluated the effects of DA application and IC stimulation on putative GABA neurons in the VTA, labeled recording sites that evinced DA and IC-induced coupling of these neurons with the anterograde tracer biotinylated dextran amine (Fig. 3D). Immunohistochemical analysis of BDA-immunoreactive (IR) sites revealed labeled neuronal clusters in the 
dorsal VTA that overlapped with the co-expression of GAD65,67 and Cx36 (Fig. 3E). The distribution of DA-sensitive, ICPSD-producing putative GABA neurons was isolated to the dorsal VTA $(n=8)$. A similar strategy was pursued in brain structures other than the VTA that showed high-density co-expression of GAD65,67 and Cx36. We found that neurons in these regions had similar discharge profiles (i.e., high firing rate, phasic activity) and spike waveform characteristics (short-duration spikes; Fig. 4). Most importantly, they produced ICPSDs similar to that produced by IC stimulation of VTA GABA neurons (Fig. 4). Neurons in these areas that produced ICPSDs were labeled with microelectrophoretic application of BDA. Immunohistochemical analysis of BDA-(IR) sites wherein ICPSDs could be elicited revealed labeled neuronal clusters in the dorsal VTA, SNr, LH, and RTN (n=30; Fig. 4), and correlated with areas containing high-density co-expression of Cx36 and GAD65,67 transcripts (Fig. 2). Despite the fact that the AH, MPA/MPO, and ventral HPC showed high-density coexpression of Cx36 and GAD65,67 transcripts (Fig. 2) we were unable to elicit ICPSDs in neurons in these areas ( $\mathrm{n}=5$ each).

We have previously demonstrated that VTA GABA neuron firing rate accelerates prior to MFB self-stimulation, but is inhibited immediately thereafter (22). The fact that VTA GABA neurons anticipate MFB self-stimulation is somewhat counterintuitive, given the assumption that VTA GABA neurons inhibit reward-related DA neurons. Indeed, working under this prevailing model, we initially assumed that IC stimulation in freely-behaving rats would be aversive, given the prevailing dogma that VTA GABA neurons inhibit DA neurons and that the pronounced enhancement of their activity and electrical coupling by IC stimulation would inhibit DA neural firing. Indeed, our initial intent was to use IC stimulation as an aversive stimulus to determine if the acceleration of VTA GABA neuron firing rate before a rewarding stimulus might be similar to that during the rat's approach to the termination of an aversive stimulus, which might suggest that VTA GABA neurons were more involved in selective attention than reward per se. However, it became surprisingly evident that rats would perform IC self-stimulation, and they typically performed at similar, if not higher, rates to that of MFB self-stimulation. Moreover, if we stimulated the MFB with a monopolar 
electrode, similar to that of other self-stimulation studies, we elicited VTA GABA neuron PSDs in a manner identical to that produced by bipolar stimulation electrodes in the IC, suggesting that VTA GABA neuron coupling, or ventral brain GABA neuron coupling, may be responsible for the BSR obtained in conventional studies utilizing MFB self-stimulation. Therefore, we postulated that electrical coupling of VTA GABA neurons might underlie BSR, at least IC self-stimulation. To determine the threshold for IC self-stimulation responding, a rate-pulse threshold study was performed in 10 rats (Fig. 5). Rats were trained on an FR-1 schedule of reinforcement with 20 IC pulses/nosepoke at $200 \mathrm{~Hz}$. Once rats reached criterion for IC self-stimulation responding (i.e., 1000 nosepokes/30 min session with little or no responding at the inactive nosepoke hole), the number of IC pulses was varied during the latter $15 \mathrm{~min}$ of each 30 min session from 20 to either 4,8,10,20,40,60, or 80 IC pulses (Fig. 5A). The rate of responding in the latter $15 \mathrm{~min}$ of the session was then expressed as a ratio of responding in the first $15 \mathrm{~min}$ of the session. The degree of responding for IC selfstimulation was characterized by an inverted U-shaped function, characteristic of doserelated responding for cocaine self-administration, of the number of pulses delivered (Fig. 5B). Thus, the rat responds proportionally less at longer pulse trains because the rewarding impact of longer pulse trains is greater.

We have recently demonstrated that dorsal VTA GABA neurons dye-couple, and that their electrical coupling is reduced by GJ blockers including mefloquine, a potent blocker of Cx36 GJ communication (25). In order to evaluate the role of VTA GABA neuron electrical coupling via GJs, we evaluated the effects of the fast-acting, reversible, but non-specific and non-selective GJ blocker quinidine, and the Cx36 GJ blocker mefloquine on IC self-stimulation response rate and threshold for responding (Fig. 6). Compared to saline control, $20 \mathrm{mg} / \mathrm{kg}$ quinidine, a dose that reduces VTA GABA neuron ICPSDs by $75 \%$ in $10 \mathrm{~min}$ for $30 \mathrm{~min}$ (20), was administered intraperitoneally $10 \mathrm{~min}$ before the session and significantly decreased overall IC selfstimulation responding $\left(\mathrm{P}<0.02, \mathrm{t}_{(2,5)}=3.6\right.$; mean saline rate $=0.52 \pm 0.11 \mathrm{~Hz}$ vs mean quinidine rate $=0.11 \pm 0.04 \mathrm{~Hz} ; \mathrm{n}=6$ each) and significantly increased the threshold for IC self-stimulation $\left(\mathrm{P}<0.002, \mathrm{t}_{(2,5)}=4.54 ;\right.$ mean saline threshold pulses $=4.5 \pm 0.62 ; \mathrm{n}=6$ 
each; Fig. 6A). Compared to vehicle control, $30 \mathrm{mg} / \mathrm{kg}$ mefloquine, a dose that reduces VTA GABA neuron ICPSDs by $50 \%$ at $24 \mathrm{hr}$ for 3 days (19), was administered intraperitoneally $24 \mathrm{hr}$ before the session did not significantly affect overall IC selfstimulation responding $\left(\mathrm{P}>0.05, \mathrm{t}_{(2,3)}=2.2\right.$; mean vehicle rate $=0.69 \pm 0.15 \mathrm{~Hz}$ vs mean mefloquine rate $=0.28 \pm 0.11 \mathrm{~Hz} ; \mathrm{n}=4$ each) but significantly increased the threshold for IC self-stimulation $\left(\mathrm{P}<0.01, \mathrm{t}_{(2,5)}=3.53\right.$; mean saline threshold pulses $=3.5 \pm 0.62$; $\mathrm{n}=6$ each; Fig. 6B). Thus, the rewarding impact of the stimulation is lessened under the influence of these GJ antagonists because stronger stimulation is required to produce a given threshold level for responding and performance. In order to determine if decreases in IC self-stimulation thresholds might be due to deficits in motor performance we evaluated response rates during the first $200 \mathrm{sec}$ of control and drug sessions. There was no difference in response rates between quinidine vs saline control $\left(\mathrm{P}>0.05, \mathrm{t}_{(2,3)}=0.03\right.$; mean saline control rate $=1.08 \pm 0.4 \mathrm{~Hz}$ vs quinidine rate $=1.1 \pm$ $0.3 \mathrm{~Hz} ; \mathrm{n}=4)$ or mefloquine vs vehicle control $\left(\mathrm{P}>0.05, \mathrm{t}_{(2,6)}=0.25\right.$; mean vehicle rate $=$ $1.63 \pm 0.7 \mathrm{~Hz}$ vs mean mefloquine rate $=1.91 \pm 0.8 \mathrm{~Hz} ; \mathrm{n}=4)$.

To further pursue our hypothesis that electrical coupling between VTA GABA neurons, and perhaps other GABA neurons outside the VTA that evince coupling with IC stimulation, we characterized the input/output parameters of VTA GABA neuron coupling in light of the prevailing theory regarding BSR. Shizgal has developed the following equation (Eq 1) for predicting BSR as a function of pulse frequency and train duration (26).

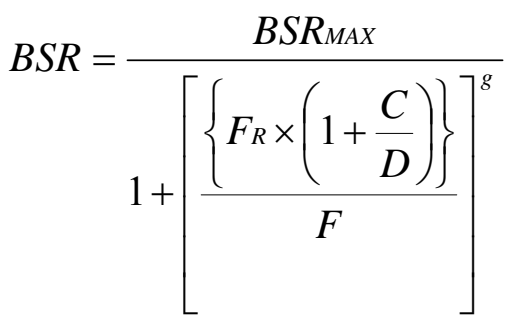

$\mathrm{C}=$ chronaxie of strength - duration function for trains, $\mathrm{D}=$ train duration, $\mathrm{F}=$ stimulation frequency, $\mathrm{F}_{\mathrm{R}}=$ frequency at which intensity of BSR is half-maximal at an infinite train duration, 
$\mathrm{g}=$ exponent of intensity growth,

$\mathrm{BSR}=$ intensity of responding for IC self-stimulation

$\mathrm{BSR}_{\mathrm{MAX}}=$ maximum intensity of responding for IC self-stimulation

Shizgal has suggested that this model and its antecedents may provide guidance for identifying the neural circuitry underlying the temporal integration of reward signals. In this regard, we have noted that this equation not only explains selfstimulation of the IC but also the discharge activity of VTA GABA neurons following stimulation via the IC. Thus, we evaluated the input/output properties of VTA GABA neuron excitability and electrical coupling by varying the stimulus parameters of IC stimulation. Since the input/output functions of IC stimulation for neurons outside the VTA were qualitatively similar to that of the VTA, we focused our analysis on VTA GABA neurons. All neurons classified as VTA GABA neurons in this study were located in the VTA, met the criteria established in previous studies for spike waveform characteristics and response to IC stimulation (18-20), and were activated and spikecoupled by microelectrophoretic DA (20). Presumed VTA GABA neurons were characterized by short-duration $(<200 \mu \mathrm{sec}$; measured at half-peak amplitude of the spike), initially negative-going, non-bursting spikes, and were identified by the following IC stimulation criteria (18): short latency (i.e., 2-5 ms) antidromic or orthodromic activation via single stimulation of the IC, and multiple spiking following high-frequency (10 pulses, $200 \mathrm{~Hz}$ ) stimulation of the IC (18-20). The mean firing rate of VTA GABA neurons recorded was $21 \pm 1.3 \mathrm{~Hz}(\mathrm{n}=85)$. Regardless of response to single stimulation, brief, high-frequency stimulation evoked ICPSDs that persisted for hundreds of msec after termination of the stimulus train (Fig. 7A). The mean number of VTA GABA neuron ICPSDs at 50\% maximum stimulus level $(200 \mathrm{~Hz}$ and 10 pulses) was $52.3 \pm 14.7(\mathrm{n}=96)$. We have previously demonstrated that the optimal frequency for generation of VTA GABA neuron ICPSDs is $200 \mathrm{~Hz}$ (18), likely due to the fact that this frequency falls within the supernormal period for antidromicallyactivated spikes (18). While holding frequency constant (i.e., $200 \mathrm{~Hz}$ ), the number of ICPSDs monotonically increased as pulse number increased (Fig. 7B,C). The threshold for activation of VTA GABA neuron ICPSDs was 4 pulses, the minimum current 
necessary at 4 pulses for activation of VTA GABA neuron ICPSDs was $0.3 \mathrm{~mA}$, and the mean number of ICPSDs/pulse was 4 as assessed by linear regression of the curves (Fig. 7D). To determine the laterality of ICPSDs, twenty one VTA GABA neurons were evaluated for response to contralateral IC stimulation, and none produced ICPSDs, suggesting that the IC input to VTA GABA neurons is restricted ipsilaterally.

Shizgal's proposed model (26) for BSR incorporates reward-growth functions based on the matching law experiments of Gallistel and Herrnstein and includes the following four properties: 1) The relationship between the train duration and the pulse frequency required to produce half-maximal BSR performance approximates a rectangular hyperbola (27); 2) At a given train duration, the subjective intensity of BSR grows as a sigmoidal function of stimulation frequency (28); 3) The sigmoidal BSR growth functions for different train durations are parallel when plotted along a logarithmic stimulation-strength axis; and 4) BSR increases as a sigmoidal function of the subjective intensity of the reward (29). While IC self-stimulation responding decreases monotonically with increasing pulse number (Fig. 5B), when this data is expressed as total current density (in microCoulombs) vs pulse number a sigmoidal response function similar to that shown by Shizgal and others is obtained (Fig. 8A). Most importantly, we find a linear relationship between IC self-stimulation and VTA GABA neuron coupling when IC self-stimulation responding is expressed as total current density delivered to the animals vs ICPSDs (Fig. 8B).

Thus, we attempted to fit our data relating BSR to VTA GABA neuron coupling to Shizgal's model. The following assumptions were made in order to relate Shizgal's model to the electrophysiology of VTA GABA neuron coupling. A frequency of 200 $\mathrm{Hz}$ was used in all experiments as it optimizes the generation of ICPSDs, due to resonance during the superexcitable period for VTA GABA neuron axonal conduction (18). Thus, with frequency held constant, we substituted stimulus intensity for frequency in the equation. This substitution is made possible because of the linear relationship between frequency and current on discharge activity. Thus, using Shizgal's 
model we obtain the following equation (Eq 2) that describes the relationship between stimulus intensity and pulse number and VTA GABA neuron ICPSDs.

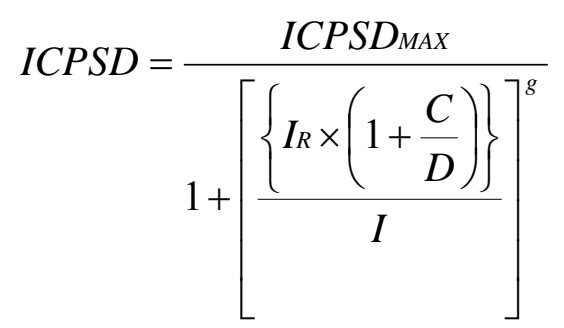

$\mathrm{I}=$ stimulation intensity,

$I_{R}=$ stimulation intensity of ICPSDs that is half-maximal at an infinite train duration, $\mathrm{g}=$ exponent of intensity growth (ICPSDs produced per pulse),

ICPSD $=$ internal capsule post-stimulus spike discharge

$\mathrm{ICPSD}_{\mathrm{MAX}}=$ maximum internal capsule post-stimulus spike discharge

For values greater than the threshold for eliciting VTA GABA neuron ICPSDs (4 pulses; Fig. 7), if we assign a value of $10 \mathrm{msec}$ as the chronaxie, representing the minimum time duration that would include 3 subthreshold pulses at $200 \mathrm{~Hz}$, and a value of 4 as the exponent of intensity growth (consistent with Shizgal's data for MFB BSR), we can effectively predict the number of ICPSDs produced as a function of stimulation current and pulse number shown in Fig. 7, which correlates directly with the strength of BSR shown in Fig. 5B.

By virtue of their wide dynamic range (i.e., high firing rate and short refractory period (18)) VTA GABA neurons, and perhaps other ventral brain GABA neurons along the rostrocaudal neuraxis, may be critical regulators of BSR. Indeed, their refractory period (i.e., $0.6 \mathrm{msec} ;(18)$ ) is within the range of the primary regulator for BSR (i.e., 0.4-1.2 msec (30)). While DA neurons are activated by MFB selfstimulation, they are not activated by IC self-stimulation, while GABA neurons appear to be activated by both, at least when MFB self-stimulation is monopolar. Indeed, a role for DA in BSR has been called into question by recent reports demonstrating that there is no DA release (31), or decreased DA release (16), during each operant response 
for MFB self-stimulation, and that GABA inhibitory projections from the VTA to the NAcc play an unappreciated role in BSR (32). Notwithstanding the prevailing dogma that GABA neurons inhibit DA neurons in the VTA, we have no direct evidence to date that this subpopulation of dorsally-located, dye-coupled, and electrically-coupled Cx36expressing GABA neurons are connected to DA neurons in the VTA. Indeed, the converse appears to be more operational, as DA excites VTA GABA neurons. Moreover, there is no correlation between simultaneously-recorded VTA GABA and DA activity in freely-behaving rats during any phase of the sleep-wake cycle or cortical activation (21). Thus, this subpopulation of VTA GABA neurons may be part of a larger network of GABA neurons that operate independently of the mesolimbic DA system. Their electrical coupling might endow them with the ability to synchronous GABA inhibitory networks to the corticolimbic system. Indeed, their antidromic activation by IC stimulation $(18,19)$, and their correlation with electrocortical activity (21) suggests that they might be involved in cortical activation, perhaps the attention to rewarding stimuli which might require fast synchronous activity between GABA neurons. The distribution of Cx36 and GAD65,67 colobeled neurons in the VTA and along the ventral brain neuraxis provides anatomical evidence that supports the physiological evidence (i.e., ICPSDs) that GABA neurons are connected electrically. Interestingly, electrically-coupled RTN GABA involved in the generation of cortical spindles (33) are coupled by IC stimulation and exhibit high-density co-expression of Cx36 and GAD65,67, suggesting that GABA neurons in the VTA and other areas along the ventral brain neuraxis may form a GABA electrical network with RTN neurons. This web of GABA neurons may explain the diffuse nature of BSR and the difficulty in interpreting lesion studies, in that self-stimulation could be effectively elicited from multiple structures in the ventral brain. This is consistent with the results of careful experiments that have failed to block BSR by lesioning the MFB and presumed rewardrelevant pathways (17). The electrical coupling between VTA GABA neurons that we have observed in vivo (20) is an order of magnitude greater than that seen for other coupled neuron types studied to date. Mainly, spike-to-spike coupling produced by DA or by afferent stimulation of the IC is 1:1. Future studies will determine electrical coupling between VTA GABA neurons identified in GAD GFP mice with membrane 
potential sensitive fluorescent dyes to directly assess the organization of GABA electrical networks in the ventral brain. Taken together with our previous findings that VTA GABA neurons are involved in cortical activation (21) and their discharge activity anticipates BSR (22), as well as our findings reported here that they compose part of a larger syncytium, or reticulum, of GABA neurons in the midbrain, thalamus, and hypothalamus linked by electrical synapses via Cx36 GJs, and that IC self-stimulation is a direct function of VTA GABA neuron electrical coupling (i.e., ICPSDs), these presumed GABA neurons appear to be critical integrators of BSR. 


\section{Figure 1}
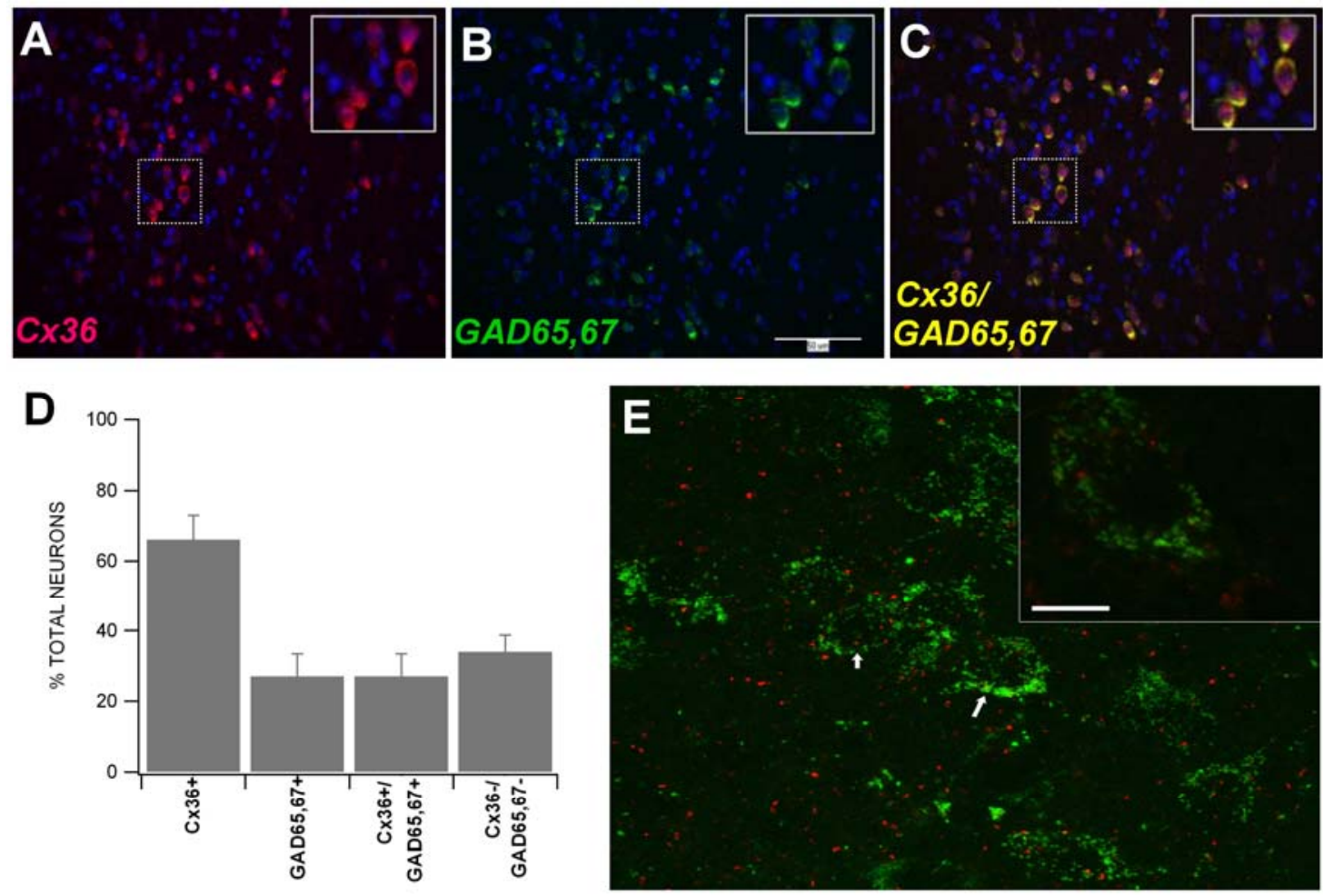

Co-expression of Cx36 and GAD65,67 in the dorsal VTA. (A-C) These are 20X magnification images of Cx36, GAD65,67 and superimposed Cx36/GAD65,67 fluorescent in-situ hybridization (FISH) in the dorsal VTA of mature rats. Cx36 and GAD65,67 were detected with CY3-labeled (red) and FITC-labeled (green) riboprobes, respectively. All images illustrate DAPI nuclear staining in blue. The insets show enlarged views of the area indicated by the dashed square on the 20X images and reveal the coexpression of Cx36/GAD65,67 in dorsal VTA neurons. Scale bars in B corresponds to all images. (D) This graph summarizes the FISH analysis of $\mathrm{Cx} 36+$, GAD65,67+, Cx36+/GAD65,67+, and Cx36-/GAD65,67- neurons in horizontal slices from 4 rat brains. Of the neurons in the dorsal VTA, $66 \%$ were $\mathrm{Cx} 36+, 27 \%$ were GAD65,67+, and 34\% were Cx36+/GAD65,67+. All neurons that were GAD65,67+ were $\mathrm{C} \times 36+$. (E) This image shows a 20X magnification image of the immunohistochemical distribution of Cx36 (red) and GAD65,67 (green) in the dorsal VTA. What do the arrows point to??? These data show that the mRNA transcripts result in expression of Cx36 and GAD65,67 protein in the dorsal VTA. 


\section{Figure 2}
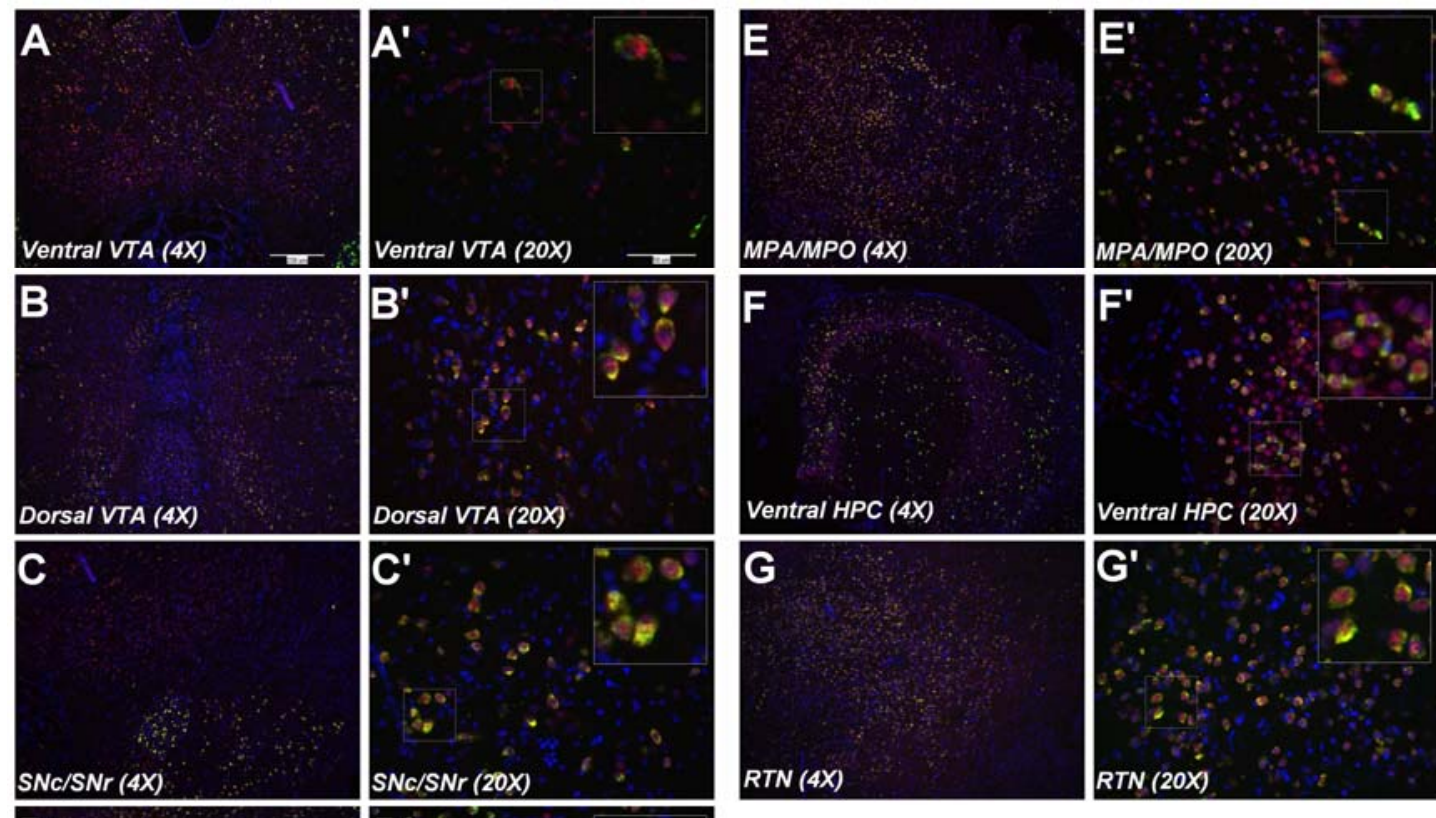

D
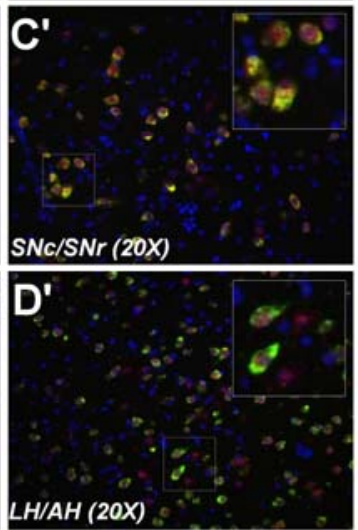

$\operatorname{RTN}(20 X)$

Co-expression of Cx36 and GAD65,67 in structures along the rostrocaudal neuraxis from the ventral tegmental area (VTA) to the reticular thalamic nucleus (RTN). (A-G) These are 4X and 20X (A'-G') magnification images of Cx36/GAD65,67 fluorescent in-situ hybridization (FISH) in the ventral VTA, dorsal VTA, substantia nigra (SN), lateral hypothalamus $(\mathrm{LH})$ /anterior hypothalamus $(\mathrm{AH})$, medial preoptic area (MPA)/medial preoptic nucleus (MPO), ventral hippocampus (HPC), and reticular thalamic nucleus (RTN). Cx36 and GAD65,67 were detected with CY3-labeled (red) and FITC-labeled (green) riboprobes, respectively. All images illustrate DAPI nuclear staining in blue. The insets show enlarged views of the area indicated by the dashed square on the 20X images and reveal the coexpression of Cx36/GAD65,67 in each respective area. Scale bars in A,A' correspond to all images. 


\section{Figure 3}
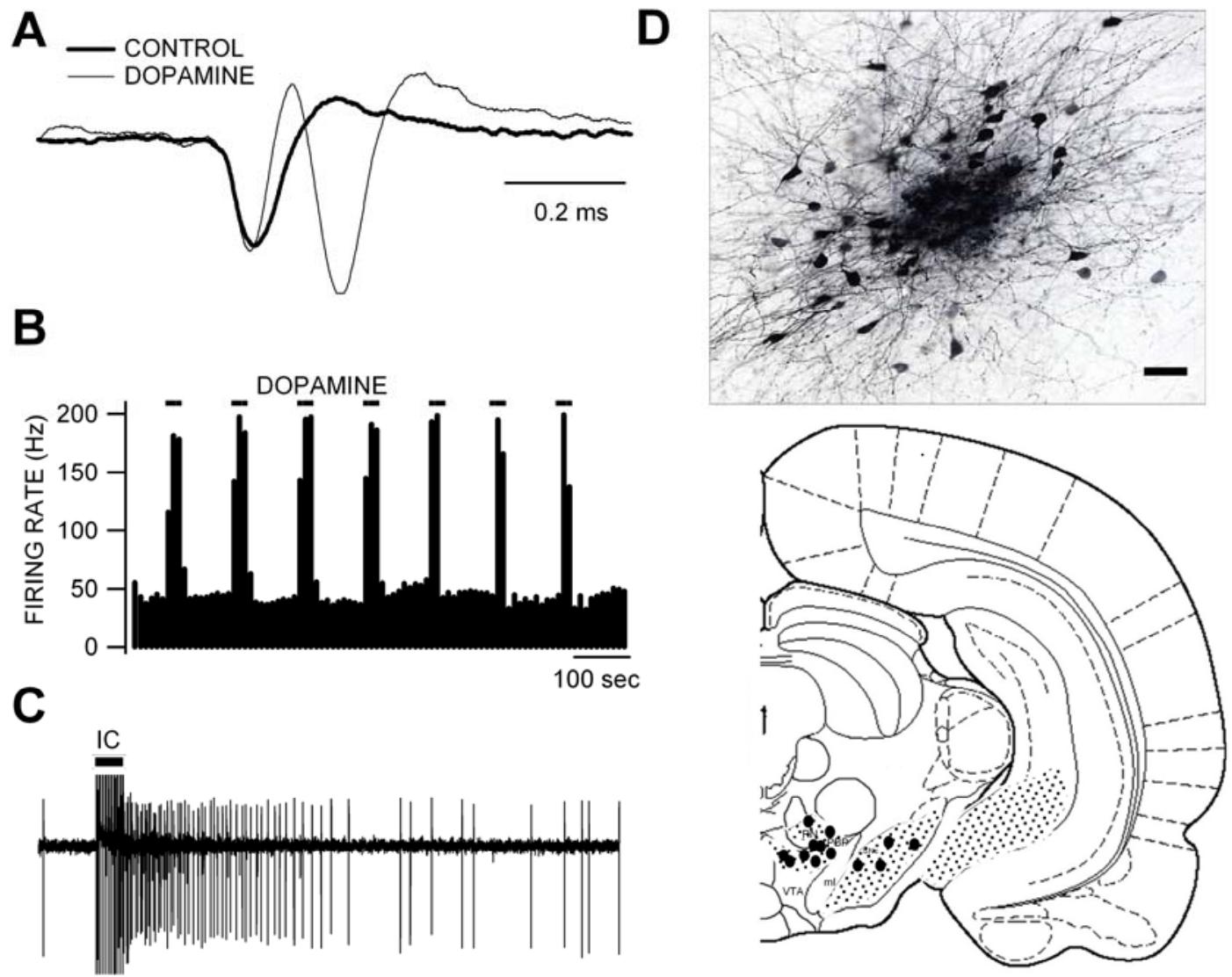

Dopamine-sensitive electrically-coupled GABA neurons are localized to the VTA and nearby structures and correlate with areas showing high expression of GAD 65,67 and Cx36. (A) These superimposed traces show unfiltered recordings of a representative VTA GABA neuron spike before (fine line) and after (heavy line) in situ microelectrophoretic application of dopamine (+20 nA). Note that VTA GABA neuron spike waveforms are characterized by an initial negative-going deflection followed by a small positive-going potential. The duration of the negative-going component of the spike waveform is approximately $100 \mu \mathrm{sec}$. Microelectrophoretic application of dopamine elicited a trailing spike couplet to the waveform. (B) This ratemeter record shows that dopamine markedly enhances the firing rate of VTA GABA neurons without any diminution in their activation by repetitive dopamine current. Despite a marked increase in firing rate with microelectrophoretic pulsing of DA current the leading spike appeared to be unaffected by dopamine. The coupled spike followed the leading spike faithfully even at dopamineevoked firing rates approaching $200 \mathrm{~Hz}$. (C) This trace shows the effects of brief, high-frequency $(200 \mathrm{~Hz}, 10$ pulses $)$ stimulation of the internal capsule (IC) on the discharge activity of a VTA GABA neuron. Note the IC-evoked poststimulus spike discharges (ICPSDs) that follow the artifact extend for hundreds of msec after the train. We have previously demonstrated that ICPSDs are sensitive to gap junction blockers (19, 20). (D) Recording sites associated with spikes that were coupled by dopamine and elicited ICPSDs were subsequently labeled by microelectrophoretic application of the anterograde tracer biotinylated dextran amine (BDA), which labels multiple neurons near the pipette. (E) This Paxinos and Watson coronal plate at $5.8 \mathrm{~mm}$ posterior to bregma shows BDA-labeling sites where GABA neurons were sensitive to dopamine and ICPSDs were elicited (filled circles). Areas showing high expression of Cx36 and GAD65,67 co-expression at this brain location are depicted in the shaded areas (see Fig. 1). Note the overlap of BDA-labeled sites and co-labeling for Cx36 and GAD65,67 in the dorsal VTA and the substantia nigra pars reticulate $(\mathrm{SNr})$. Abbreviations: $\mathrm{ml}$ - medial lemniscus; $\mathrm{PBP}$ - parabrachial pigmented nucleus; $\mathrm{RN}$ - red nucleus; $\mathrm{SNr}$ - Substantia Nigra pars reticulata; VTA - ventral tegmental area. 


\section{Figure 4}
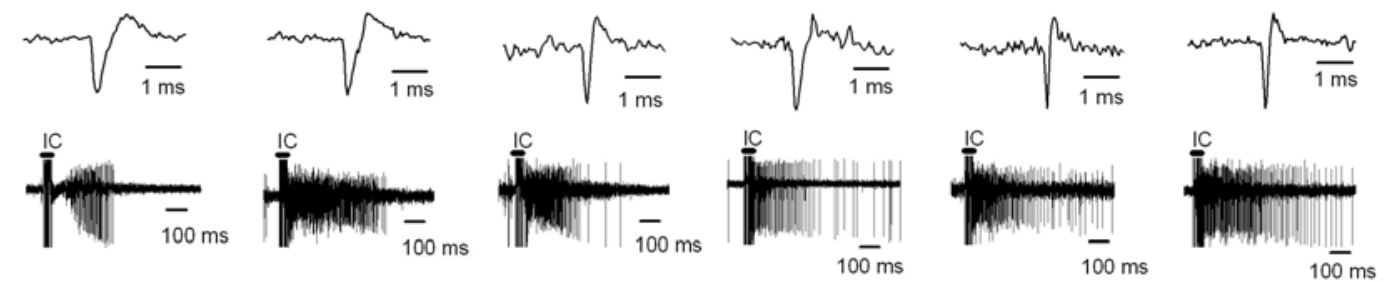

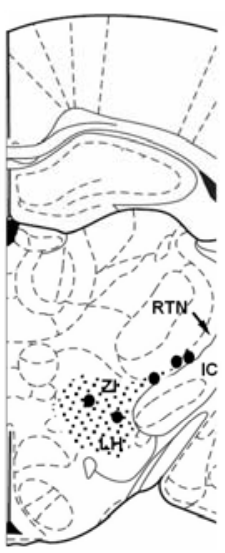

$-2.8$

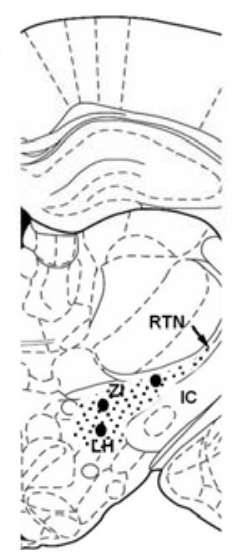

$-3.3$

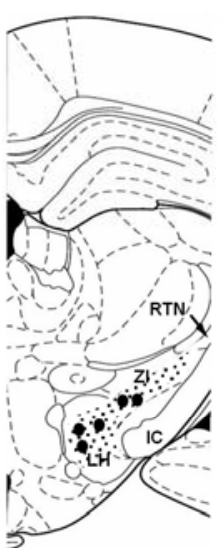

$-3.8$

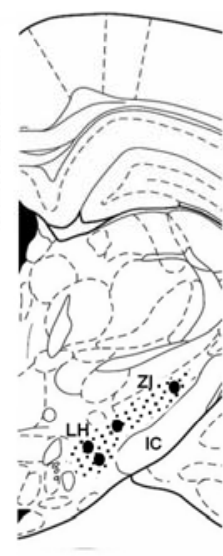

$-4.3$

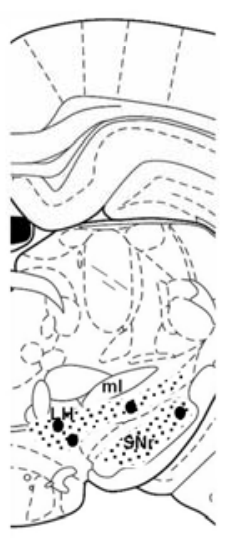

$-4.8$

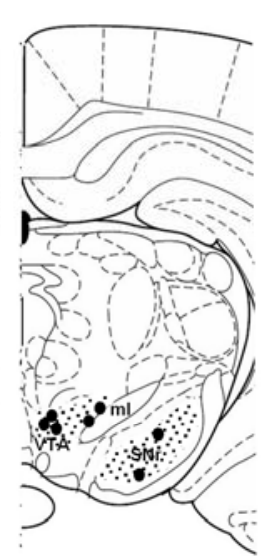

$-5.3$

Coupling of neurons along the rostrocaudal ventral neuraxis at locations demonstrating co-expression of Cx36 and GAD65,67 transcripts. Spike waveforms and IC-evoked post-stimulus spike discharges (ICPSDs) are shown above Paxinos and Watson coronal plates at locations posterior to bregma $(-2.8$ to $-5.3 \mathrm{~mm})$ in $0.5 \mathrm{~mm}$ serial sections. Brief, high-frequency (10 pulses, $200 \mathrm{~Hz}$ ) stimulation elicited ICPSDs at locations along the rostrocaudal neuraxis from the IC to the VTA. The traces above show spike waveforms at stereotaxic locations where ICPSDs were elicited. Biotinylated dextran amine (BDA) was iontophoretically applied from the recording pipette at sites producing ICPSDs. Filled circles indicate locations where BDA-labeled cells were detected (see BDA-labeled neurons in Fig. 3). Shaded areas indicate high density of coexpression of Cx36 and GAD65,67 (see Fig. 2). BDA-labeled cells corresponding to locations where ICPSDs could be elicited were found in areas that also showed highdensity co-expression of Cx36 and GAD65,67. The overlap extended from the midbrain through the lateral hypothalamus to the midbrain. Abbreviations: LH - lateral hypothalamus, $\mathrm{ml}$ - medial lemniscus; $\mathrm{PBP}$ - parabrachial pigmented nucleus; $\mathrm{RN}$ - red nucleus; reticular thalamic nucleus (RTN) SNr - Substantia Nigra pars reticulata; VTA ventral tegmental area; ZI - zona incerta. 


\section{Figure 5}

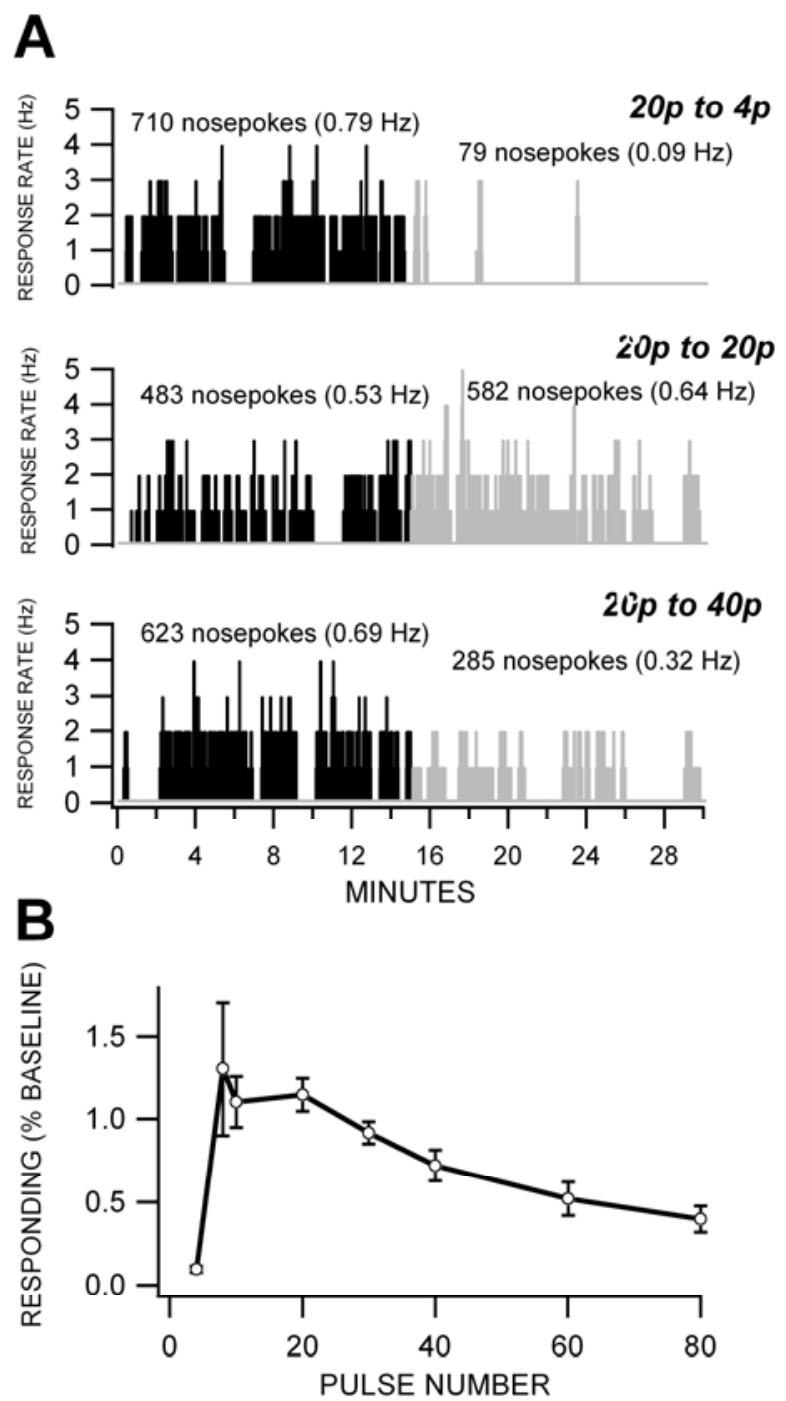

The threshold for VTA GABA neuron ICPSDs is the threshold for IC self-stimulation responding. Rats were allowed ad libitum access to a nosepoke hole that triggered a constant current stimulator that delivered stimulus pulses to the IC (20 pulses; $200 \mathrm{~Hz}$; half-maximum stimulus level). Once rats reached criterion for responding (1000

nosepokes $/ 30 \mathrm{~min}$ session; $0.56 \mathrm{~Hz}$ ) at the active nosepoke responding typically stabilized to throughout the $30 \mathrm{~min}$ session and between sessions, with little or no responding at the inactive nosepoke hole. (A) These histograms show IC self-stimulation responding for a typical rat with average responding during 3 separate sessions (top, middle, bottom) wherein the IC pulses were changed from 20 pulses to the value indicated in each histogram. At 15 min into the 30 min session the number of stimulus pulses delivered to the IC was switched from 20 to 4 pulses (top; black to grey on the graph), 20 to 20 pulses (middle), and 20 to 40 pulses (bottom). The rate of responding for the 20-4 pulse transition transiently decreased and then subsided markedly. The rate of responding for the $20-20$ pulse transition produced a mild increase in responding. The rate of resonding for the 20-40 pulse transition moderately decreased responding $(\sim 50 \%)$. (B) This graph shows the rate of IC self-stimulation responding as a function of pulse number. Values are expressed as the grand average across rats of the ratio of the average rate of responding after the transition from 20 pulses to the number on the abscissa (second half of the session) vs the average rate of responding with 20 pulses (first half of the session). Compared to the response rate at 20 pulse IC self-stimulation, responding was characterized by an inverted U-shaped curve with threshold at 4-5 pulses, variable responding from 5-10 pulses, and decreased responding to 80 pulses. 
Figure 6
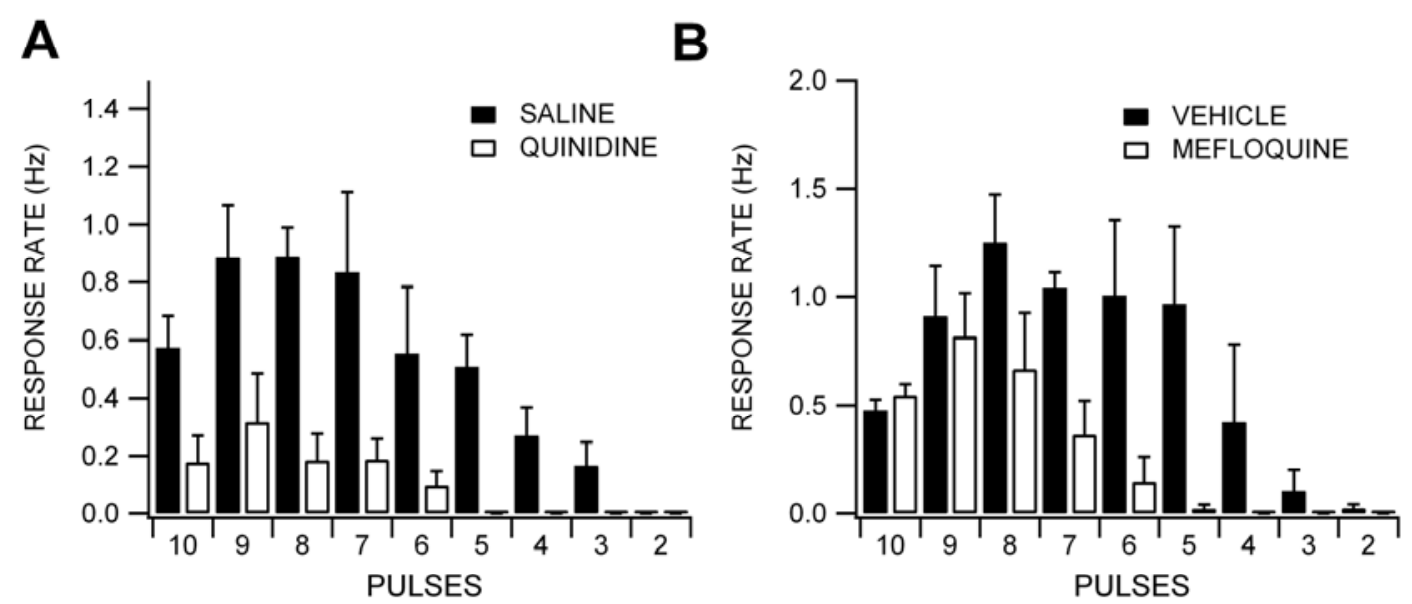

Gap junction blockers raise the threshold for IC self-stimulation responding. Rats were trained to perform IC self-stimulation to criterion on 20 IC pulses. To study the effects of quinidine and mefloquine on threshold for responding we systematically decreased the pulse number within a session for a set number of responses at each pulse level. (A) This graph shows the effects of intraperitoneal administration of the GJ blocker quinidine vs saline on the pulse threshold for IC self-stimulation. Quinidine $(20 \mathrm{mg} / \mathrm{kg})$ was administered 10 min before the session. Quinidine significantly decreased overall responding with affecting initial performance for $20 \mathrm{IC}$ pulses at the onset of the session (data not shown--see text). Note that the IC pulse threshold for IC self-stimulation responding was raised by quinidine. (B) This graph shows the effects of intraperitoneal administration of the Cx36 GJ blocker mefloquine vs DMSO vehicle on the pulse threshold for IC self-stimulation. Mefloquine $(30 \mathrm{mg} / \mathrm{kg}$ ) was administered $24 \mathrm{hr}$ before the session. Mefloquine decreased overall responding without affecting initial performance for $20 \mathrm{IC}$ pulses at the onset of the session (data not shown-see text). Note that the IC pulse threshold for IC self-stimulation responding was raised by mefloquine. 


\section{Figure 7}
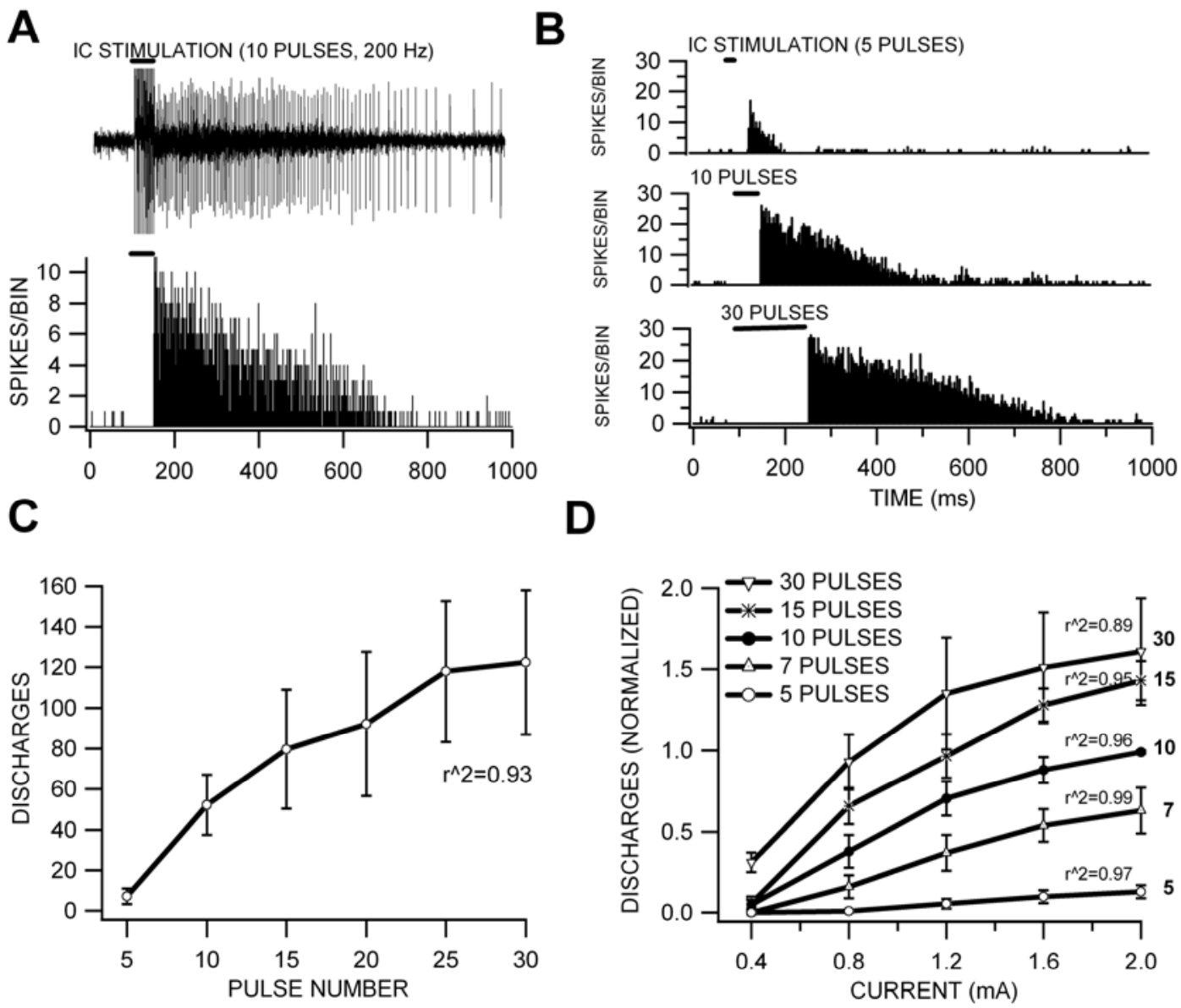

Input/output functions of IC-evoked post-stimulus spike discharges (ICPSDs):

Monotonic function of pulse number and stimulus intensity: (A) The inset shows a representative extracellular recording of a VTA GABA neuron during high-frequency stimulation (10 pulses, $200 \mathrm{~Hz}$ ) of the internal capsule (IC). VTA GABA neuron spikes not only accompany each IC stimulation pulse, but are elicited for hundreds of msec after the stimulus train has ended, hence the term "IC-evoked post-stimulus spike discharges" or ICPSDs. The peri-stimulus histogram shows the average of 12 IC stimulations producing ICPSDs. (B) These representative peri-stimulus spike histograms demonstrate that VTA GABA neuron ICPSDs increase with increasing pulse number. (C) This graph summarizes the effects of pulse number on ICPSDs. Fitting these data with linear regression analysis reveals a slope of approximately 4, indicating that 4 discharges occur for every pulse. Moreover, a threshold of 4-5 pulses at half-maximum current intensity is needed to induce discharges. (D) This graph summarizes the effects of pulse number and current on VTA GABA neuron ICPSDs. The goodness of linear fit is shown next to each of the pulse \# isobars. 


\section{Figure 8}

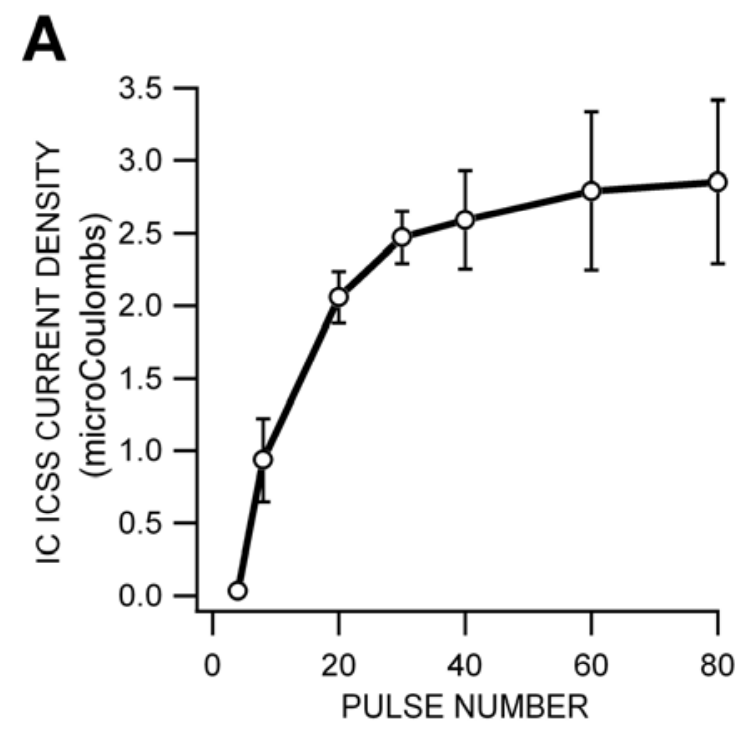

B

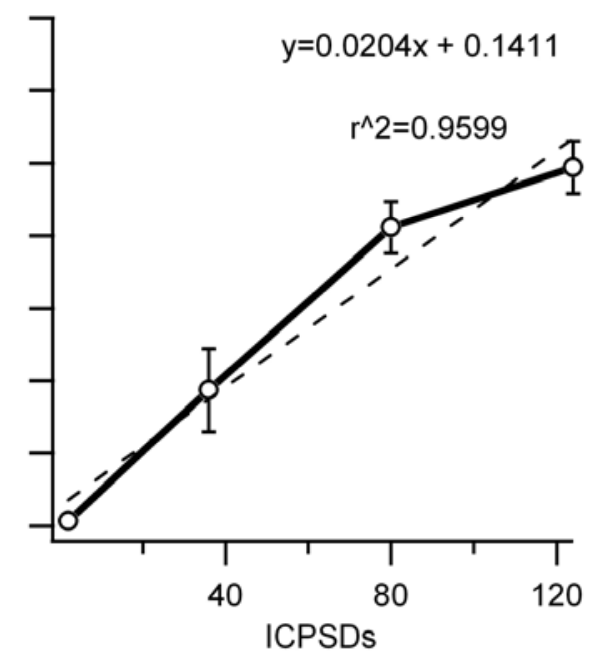

IC self-stimulation responding is a function of coupling between VTA GABA neurons.

(A) The data in Fig. 5B for IC self-stimulation responding as a function of pulse number is expressed here as a function of total current density (in microCoulombs) delivered in a self-stimulation session. Total current density was the product of IC selfstimulation responding times the pulse duration times the current. Note that the threshold remains at 4 pulses, but responding saturates at pulse numbers above 40 . (B) This graph shows the relationship between IC self-stimulation current density and the number of VTA GABA neuron ICPSDs produced by a given pulse number. The goodness of linear fit is shown next to the plot, and the equation describing the line is superimposed on the data. There is a strong correlation between VTA GABA neurons ICPSDs and the total current current density delivered during IC self-stimulation. 


\section{METHOD}

\section{Animal subjects}

Seventy-two male Wistar rats (Charles River Laboratory, Hollister, CA) weighing 250 - 450g. were used in the in vivo and in vitro experiments in this study. They were housed individually with ad libitum access to food and water, and maintained on a reverse $12 \mathrm{hr}$ light/dark cycle (off 10:00, on 22:00). Animal care, maintenance and experimental procedures were in accordance with the Brigham Young University Animal Research Committee. Adequate measures were taken to minimize pain or discomfort to the animals during the surgical procedures and behavioral testing.

Single-unit activity in anesthetized rats

Extracellular potentials in anesthetized rats were recorded by a single $3.0 \mathrm{M}$ KCl-filled micropipette (5-10 M $\Omega ; 1-2 \mu \mathrm{m}$ inside diameter), cemented 20-40 $\mu \mathrm{m}$ distal to a 4-barrel micropipette (30-80 $\mathrm{M} \Omega$ resistance), and amplified with an Axoprobe-1A microelectrode amplifier/headstage (Axon Instruments, Union City, CA). Microelectrode assemblies were oriented into the VTA [from bregma: 5.6-6.5 posterior (P), 0.5-1.0 lateral (L), 7.0-8.5 ventral (V)] with a piezoelectric microdrive (Burleigh, Fishers, NY). Single-unit activity was filtered at $1-3 \mathrm{kHz}(-3 \mathrm{~dB})$ for "filtered" recordings and 0.1-10.0 kHz for "unfiltered" recordings and displayed on analog and digital oscilloscopes. Square-wave constant current pulses (50-1000 $\mu \mathrm{A} ; 0.15 \mathrm{msec}$ duration; average frequency, $0.1 \mathrm{~Hz}$ ) were generated by an IsoFlex isolation unit controlled by a MASTER-8 Pulse Generator (AMPI, Israel) or by computer. The internal capsule (IC) (-2.5 AP, 2.5-3.0 ML, 5.0-6.5 V) stimulated with insulated, bipolar stainless steel electrodes. We evaluated spikes that had greater than 5:1 signal-to-noise ratio. Extracellularly recorded action potentials were discriminated with a Mentor N750 (Minneapolis, MN), Fintronics WDR-420 (Orange, CT), or WPI -121 (Sarasota, F1) spike analyzer and converted to computer-level pulses. Single-unit potentials, discriminated spikes, and stimulation events were captured by National Instruments NB-MIO-16 digital I/O and counter/timer data acquisition boards (Austin, TX) in Macintosh-type computers. Potentials were digitized at $20 \mathrm{kHz}$ and 12 -bit voltage resolution. 


\section{Collision testing}

The criteria for antidromicity were: 1) the lack of an EPSP preceding the spike (seen best in intracellular recordings); 2) near-constant latency at threshold stimulus levels; 3) faithful following of 5 pulses at $200 \mathrm{~Hz}$ and, most importantly, 4) collision between spontaneously-occurring and IC-evoked spikes. Collision tests were performed by triggering the stimulator with a spontaneous spike and adjusting the spontaneous spike to driven spike interval such that stimulation of the cell's axon failed to produce a spike that reached the somatic recording electrode. The absolute refractory period $r$ of VTA non-DA neurons was calculated by the following formula: $r=I-2 L$, where $I$ is the interval from the ogive of the spontaneous spike (the fastest falling edge; usually about half peak amplitude of the spike) to the ogive of the driven spike and $L$ is the latency from the stimulus artifact to the ogive of the driven spike. We accomplished this by setting $I$ at $2 \mathrm{~L}$ where $100 \%$ extinction of the driven spike was evident at $2 \mathrm{X}$ threshold stimulus level, and by increasing $I$ in $0.2 \mathrm{msec}$ increments until a driven spike occurred.

\section{Characterization of VTA GABA neurons for electrophysiological studies}

We have previously described the electrophysiological, neurochemical, and ultrastructural characteristics of VTA GABA neurons in anesthetized (3) and freelybehaving rats $(1,33)$. In brief, VTA GABA neurons represent a homogeneous population of rapid-firing neurons that project to corticolimbic structures and receive reciprocal excitatory input from these structures. All neurons classified as presumed VTA GABA neurons in this study were located in the VTA and met the criteria established in previous freely-behaving rat studies for spike waveform characteristics and response to afferent input $(1,33)$. Presumed VTA GABA neurons were characterized by short-duration $(<500 \mu \mathrm{sec}$ measured at half-peak amplitude), negative-going, non-bursting spikes (as determined from unfiltered recordings), and were identified by the following IC stimulation criteria: short latency (i.e., $<3.0 \mathrm{msec}$ ) antidromic or orthodromic activation via single stimulation of the IC, or multiple 
orthodromic spiking following high-frequency stimulation of the IC (10 pulses, 200 $\mathrm{Hz})$.

\section{ICSS behavior}

Rats were given at least one week to recover following cranial implant surgery, and were habituated by daily handling. All self-stimulation sessions took place in sound-attenuated chambers measuring $40.5 \mathrm{~cm}$ wide X $24 \mathrm{~cm}$ deep X $38 \mathrm{~cm}$ high. The commutator was suspended above the operant box and the headstage cable assembly was connected to the commutator. The commutator was also connected to a patch box that facilitated monitoring of the 4 recording electrodes and the stimulating electrodes in the IC. Two small holes drilled in opposite walls of the long-side of the operant box were equipped with infrared photocell beams. Each break of the beam in the active hole by a nosepoke delivered a train of stimuli to the IC (150 $\mu \mathrm{sec}$ pulse width, 10-80 pulses, $200 \mathrm{~Hz},+0.1-1.0 \mathrm{~mA}$ constant current; AMPI Master-8 (Jerusalem, Israel) pulse generator and constant current isolation unit). No stimulation accompanied nosepoke to the inactive hole; however, all responses were monitored. Rats were originally trained on a FR-1 schedule of reinforcement at 20 pulses, $0.5 \mathrm{~mA}$, and $200 \mathrm{~Hz}$. This paradigm was used only for the first two training sessions. The rats were then moved to 10 pulses, $0.6 \mathrm{~mA}$, and $200 \mathrm{~Hz}$ again on a FR-1 schedule of reinforcement. The criterion for responding was 1000 minimum pokes in a 30 minute session, with little or no responding on the inactive nose poke hole, which was monitored during each session. We determined threshold for ICSS responding by reducing the number of pulses received in the train by one, every 100 times the rat poked.

\section{Histology_Biotinylated dextran amine labeling}

At the termination of acute recordings, to mark DA-sensitive sites wherein VTA GABA neuron ICPSDs were elicited, and to provide morphological information on recorded neurons, $5 \%$ biotinylated dextran amine (BDA; 10,000 MW; Molecular Probes) in $1 \mathrm{M} \mathrm{KCl}$ was injected iontophoretically through one of the 4 micropipette barrels whose interior was coated with Sigmacote ${ }^{\mathrm{TM}}$ lubricant to prevent sticking. Current was applied through a Midgard or AMPI Iso-flex (Jerusalem, Israel) constant- 
current isolation source at $1-5 \mu \mathrm{A}$ using a $7 \mathrm{sec}$ ON/7 sec OFF cycle for 10 minutes. The pipette was left in situ for a minimum of $10 \mathrm{~min}$. The rat was given an overdose of halothane (5\%) and transcardially perfused with heparinized saline, followed by $4 \%$ paraformaldehyde $/ 0.2 \%$ glutaraldehyde in $0.1 \mathrm{M}$ phosphate buffer ( $\mathrm{PB}, \mathrm{pH} 7.4)$. The brains were removed and equilibrated in $30 \%$ sucrose in PB, quick-frozen, and then cut coronally into $0.1 \mathrm{M}$ PB saline (PBS, $\mathrm{pH} 7.4$ ) on a freezing microtome. BDA was visualized by incubating for a minimum of 3 hours (or as long as overnight) in avidinbiotin peroxidase complex (ABC kit, Vector Labs) as per kit protocol, then rinsed 3 times in PBS. Visualization was accomplished by rinsing tissue in $0.1 \mathrm{M}$ imidazoleacetate buffer (IAc, pH 7.3), then developing with $0.05 \%$ DAB, $2 \%$ nickel ammonium sulfate, $0.005 \% \mathrm{H}_{2} \mathrm{O}_{2}$ in IAc. After processing for BDA, sections were mounted on slides, counterstained with neutral red, dehydrated, and coverslipped with Permount ${ }^{\mathrm{TM}}$ (Fisher) under glass coverslips. At the termination of the chronic recordings, electrolytic lesions were made by passing current ( $\pm 3 \mathrm{~mA}, 5 \mathrm{sec}$ duration) with a constant current stimulus isolation unit (Grass Instruments, Quincy, Mass) through the recording electrodes during deep anesthesia. The animals were subsequently administered a lethal dose of halothane or pentobarbital and the brains were removed and preserved as described above. The brains were frozen and sectioned in a cryostat for inspection of the lesion site.

\section{Histology—fluorescent in-situ hybridization.}

Horizontal brain slices $250 \mu \mathrm{m}$ were prepared with a vibratome. Slices were covered with OCT, flash-frozen in isopentane and sectioned $(20 \mu \mathrm{m})$ with a cryostat. Sections were processed for FISH as described in Guzowski, J. F., McNaughton, B. L., Barnes, C. A. \& Worley, P. F. (1999) Nat. Neurosci. 2, 1120-1124. Images were acquired with a Nikon TE2000U epifluorescence microscope equipped with a spinning disk confocal imaging system as described (Guzowski JF, Miyashita T, Chawla MK, Sanderson J, Maes LI, Houston FP, Lipa P, McNaughton BL, Worley PF, Barnes CA. Recent behavioral history modifies coupling between cell activity and Arc gene transcription in hippocampal CA1 neurons. Proc Natl Acad Sci U S A. 2006 Jan 24;103(4):1077-82). Antisense digoxigenin-labeled connexin 36 and fluorescein- 
labeled GAD 65/67 cRNA riboprobes were hybridized overnight at 56oC and detected using a commercial biotin tyramide amplification kit with streptavidin CY3 and a FITC tyramide amplification kit (DirectFISH; PerkinElmer Life Sciences), respectively.

Sections were then counterstained with DAPI (Molecular Probes, Invitrogen, Carlsbad, CA). The GAD 65/67 riboprobes were generously provided by Dr. John Guzowski (Center for the Neurobiology of Learning and Memory, University of California, Irvine). Plasmid DNA for preparation of the connexin 36 riboprobe was kindly provided by Dr. Viviana Berthoud (Department of Pediatrics, University of Chicago). The plasmid, a $653 \mathrm{bp}$ DNA fragment containing part of the coding sequence for mouse connexin 36 inserted in pGEM-T Easy (Promega, Madison, WI), was linearized with NcoI and the FISH probe was generated using SP6 RNA polymerase.

To demonstrate protein colocalization, $30 \mu \mathrm{m}$ thick horizontal rat brain sections were processed immunohistochemically for 1) GAD67-IR (Chemicon monoclonal, 1:800 dilution) and 2) Cx36-IR (Zymed rabbit polyclonal, 1:500). Tissue was preincubated in $3 \% \mathrm{H}_{2} \mathrm{O}_{2}$ and $1 \%$ Triton X-100 (TX) for $15 \mathrm{~m}$, then rinsed for $5 \mathrm{~m} 3$ times in PB. Tissue was then incubated in primary antibody solution $(5 \%$ normal horse serum, $0.3 \% \mathrm{TX}$, in $0.1 \mathrm{M} \mathrm{PB}$ ). After overnight incubation, tissue was rinsed as before, then placed in HRP-conjugated goat anti-rabbit (Jackson Immuno, 1:600) and biotinylated goat anti-mouse (Jackson Immuno, 1:600) secondary antibodies (5\% normal serum, $0.1 \% \mathrm{TX}$ in $0.1 \mathrm{M} \mathrm{TBS}$ ). After incubating $90 \mathrm{~min}$, tissue was again rinsed 3 times in $\mathrm{PB}$. It was then reacted with AlexaFluor 488-conjugated tyramide and $0.003 \% \mathrm{H}_{2} \mathrm{O}_{2}$ in $\mathrm{PB}$ for 15 minutes, rinsed once in $\mathrm{PB}$ with $3 \% \mathrm{H}_{2} \mathrm{O}_{2}$ to quench excess $\mathrm{HRP}$, then 3 times in $\mathrm{PB}$, and finally placed in AlexaFluor 594-conjugated streptavidin (Molecular Probes, 1:300) for $60 \mathrm{~min}$. Tissue was rinsed thoroughly, then mounted on chrome-alum dipped glass slides. All slides were air-dried, dehydrated through successive ethanols, cleared in xylene, and then coverslipped in DEPEX.

\section{Image Analysis}

The quantification of images acquired from the dorsal VTA was done using MetaMorph software (Universal Imaging Corporation, West Chester, PA). Images of 
the dVTA were taken lateral to the caudal linear raphe nu (CLi) in four horizontal sections representing three different animals as previously described in our methods. Manual cell counts were done on putative neurons from the median planes of the Zstack images (representing $20 \%$ of the stack thickness). Neurons were outlined and identified as either negative, Connexin 36 positive, Gad65/67 positive or as positive for both. Putative glial cells were identified and excluded based on their small size and bright, uniform nuclear counterstaining with DAPI as described by Vazdarjanova et. al. The results were expressed as the percentage of total positive cells for each riboprobe. Image capture of Cx36/GAD67 immunolabeled cells was performed with a Nikon laser scanning confocal microscope. Images of relevant areas were captured as $12 \mu \mathrm{m}$ thick z-stacks made up of $1 \mu \mathrm{m}$ optical thickness individual images, and merged using Volocity software.

Vazdarjanova, A., McNaughton, B. L., Barnes, C. A., Worley, P. F. \& Guzowski, J. F. (2002) J. Neurosci. 22, 10067-10071

\section{Drug delivery}

For systemic administration of drugs in vivo, mefloquine was solubilized in $1 \%$ Tween 80 in distilled water and delivered intraperitoneally. Quinidine was solubilized in saline and delived intravenously. Systemic drug responses were compared to vehicle (1\% Tween80) and saline injections. Mefloquine was a gift from the Walter Reed Army Institute of Research (Silver Spring, MD).

\section{Analysis of responses}

Waveforms, discriminated spikes and stimulation events were processed with National Instruments LabVIEW and IGOR Pro software (Wavemetrics, Lake Oswego, OR). Spike durations were measured by orienting cursors on the waveforms at halfmaximum peak amplitude of the negative-going spike. Ratemeter records were analyzed by orienting cursors on the ratemeter records to integrate the average firing rate of VTA GABA neurons over 5 min epochs before drug, during drug, and following 
recovery. Peri-stimulus spike histograms (PSHs) were constructed for determinations of the number of VTA GABA neuron ICPSDs. The histograms were normalized to number of internal capsule stimulations before and after drug treatment (12 stimulation trains at $10 \mathrm{sec}$ intervals, $1 \mathrm{sec}$ epoch, $2 \mathrm{~ms}$ bin width). The number of driven spikes following internal capsule stimulation was determined by rectangular integration using IGOR Pro software. As the number of discharges varied across neurons within each animal and across animals, we integrated spikes on PSHs falling in bins immediately after the stimulation epoch and extending to a point on the PSH where the discharges appeared to be just above the floor of spontaneous activity (range between 250-600 ms beyond the stimulus artifact). To further reduce variability across treatment groups, we standardized ICPSDs to percent control. The results for control and drug treatment groups were derived from calculations performed on spontaneous firing rate and PSHs and expressed as means \pm S.E.M. The results were compared before and after drug treatment using the paired two-sample for means t-test, and single factor ANOVA, for comparisons between groups of unequal sample size. The criterion of significance was set at $\mathrm{p}<0.05$. 


\section{REFERENCES}

1. J. Olds, P. Milner, J Comp Physiol Psychol 47, 419 (Dec, 1954).

2. $\quad$ M. Miguelez, C. Bielajew, Rev Neurosci 15, 47 (2004).

3. $\quad$ R. A. Wise, P.-P. Rompre, Ann. Rev. of Psychol. 40, 191 (1989).

4. G. Hernandez et al., Behav Neurosci 120, 888 (Aug, 2006).

5. C. R. Gallistel, M. Leon, B. T. Lim, J. C. Sim, M. Waraczynski, Behav Neurosci 110, 766 (Aug, 1996).

6. O. Lindvall, A. Bjorklund, Acta Physiol. Scand. Suppl. 412, 1 (1974).

7. C. Y. Yim, G. J. Mogenson, Brain Research 181, 301 (1980).

8. H. Maeda, G. J. Mogenson, Brain Research 197, 365 (1980).

9. D. C. German, M. Dalsass, R. S. Kiser, Brain Res. 181, 191 (1980).

10. C. Bielajew, P. Shizgal, J. Neurosci. 6, 919 (1986).

11. P. G. Guyenet, G. K. Aghajanian, Brain Res. 130, 69 (1978).

12. C. R. Gallistel, in The physiological basis of memory J. A. Deutsch, Ed. (Academic, New York, 1983) pp. 269-349.

13. P. Shizgal, I. Kiss, C. Bielajew, in The neural basis of feeding and reward B. G. Hoebel, D. Novin, Eds. (Haer Institute, Brunswick, 1982) pp. 419-429.

14. R. A. Wise, Pharmacology, Biochemistry and Behavior 13, 213 (1980).

15. R. A. Wise, Annu. Rev. Neurosci. 19, 319 (1996).

16. P. A. Garris et al., Nature 398, 67 (1999).

17. J. M. Simmons, R. F. Ackermann, C. R. Gallistel, J Neurosci 18, 8515 (1998).

18. S. C. Steffensen, A. L. Svingos, V. M. Pickel, S. J. Henriksen, J. Neurosci. 18, 8803 (1998).

19. D. W. Allison et al., Synapse 60, 20 (2006).

20. S. H. Stobbs et al., J Pharmacol Exp Ther 311, 282 (2004).

21. R. S. Lee, S. C. Steffensen, S. J. Henriksen, J. Neurosci. 21, 1757 (2001). 
22. S. C. Steffensen, R.-S. Lee, S. H. Stobbs, S. J. Henriksen, Brain Res. 906, 190 (2001).

23. J. Degen et al., J Comp Neurol 473, 511 (Jun 7, 2004).

24. X. B. Liu, E. G. Jones, J Comp Neurol 466, 457 (Nov 24, 2003).

25. S. J. Cruikshank et al., Proc Natl Acad Sci U S A 101, 12364 (Aug 17, 2004).

26. B. Sonnenschein, K. Conover, P. Shizgal, Behav Neurosci 117, 978 (Oct, 2003).

27. C. R. Gallistel, J Comp Physiol Psychol 92, 977 (Dec, 1978).

28. C. R. Gallistel, M. Leon, Behav Neurosci 105, 913 (Dec, 1991).

29. R. J. Herrnstein, J Psychiatr Res 8, 399 (Aug, 1971).

30. J. S. Yeomans, Physiol. Behav. 15, 593 (1975).

31. Z. L. Kruk et al., J Neurosci Methods 79, 9 (1998).

32. J. F. Cheer, M. L. Heien, P. A. Garris, R. M. Carelli, R. M. Wightman, Proc Natl Acad Sci U S A 102, 19150 (Dec 27, 2005).

33. P. Fuentealba, M. Steriade, Prog Neurobiol 75, 125 (Feb, 2005). 


\section{Education}

- Brigham Young University:

- MS: Neuroscience (12/06)

- BS: Neuroscience, 3.8 GPA (12/02)

- Utah Fire and Rescue Academy:

- EMT-I, \#016216, Exp: 09/30/08

- Firefighter I and Hazmat Awareness

- NFPA Wildland Firefighter I and NWCG Firefighter II

\section{Work and Service Experience}

- Neuro-Electrophysiology Lab (09/02-present): Researching the neural mechanisms behind pleasure and addiction; as a lab assistant my duties include;

- Manage and organize groups of students/projects

- Surgical procedures

- Measuring the physiological response of cells to various stimuli

- Firefighter, EMT (01/00-present): North Fork Fire Department, Sundance Utah;

- Captain in the department: (9/2001-9/2002)

- The department works on a volunteer basis where I commit ten hours weekly

- My time at the department is spent in training, creating a positive relationship with the public, cleaning the station, and responding to both fire and EMS calls

- Biophysical Lab (05/03-08/05): Researching the mechanisms underlying neural secretions.

- Measure ion conductance across lipid bilayers

- Isolate synaptic vesicles from biological tissue

- Molecular Biology Lab (03/02-04/03): Elucidating the genetic diversity of Colorado native fish; as lab assistant my duties include;

- Isolating and sequencing raw DNA

- Analyzing the genetic code for functional mutations between species.

- References available upon request

\section{Contact Information}

- Email: matthew_lassen@byu.edu 


\section{Publications}

Papers

- $\quad$ Stobbs, S.H., Ohran, A.L., Lassen, M.B., Allison, D.W., Brown, J.E. and Steffensen, S.C., Ethanol suppression of ventral tegmental area GABA neuron electrical transmission involves NMDA receptors, J Pharmacol Exp Ther, 311 (2004) 282-289

Abstracts

- Lassen, M.B., Gunderson, S.H., Eysser, M.G., Stobbs, S.H., Ray, A.P., Henriksen, S.J., and Steffensen, S.C. Brain stimulation reward is integrated by a network of electrically-coupled GABA neurons in the midbrain tegmentum. Soc. Neurosci, Absts (2005) 31: 779.10

- S.C. Steffensen, A.J. Ohran, J.E. Brown, M.B. Lassen, and S.H. Stobbs. Ethanol suppression of VTA GABA neuron ephaptic transmission is mediated by NMDA receptors. Alcoholism: Clin. Exp. Res. (2004) 28(5) 132A

- Steffensen, S.C., Stobbs, S.H., Allison, D.W., Lassen, M.B., Brown, J.E., Henriksen, S.J. Ventral tegmental area GABA neurons form a network of dopamine-sensitive electrical synapses: Role in brain stimulation reward. Soc. Neurosci. Absts (2003) 29: 679.4

- Steffensen, S.C., Stobbs, S.H., Allison, D.W., Brown, J.E., Lassen, M.B., Lee, R.S., and Henriksen, S.J. Differential adaptation of ventral tegmental area GABA neurons by ethanol and barbiturates. Alcoholism: Clin. Exp. Res. (2003) 27(5) 56A

Grants on which I have been coPI

- Steffensen, S.C. Student Mentoring Grant 2004. Neuropsychology laboratory. Principal Investigator. Funded by BYU ORCA $(\$ 20,600)$

- Steffensen, S.C. Student Mentoring Grant 2003. Neuropsychology laboratory. Principal Investigator. Funded by BYU ORCA $(\$ 19,600)$ 